\title{
RhoA/Rho kinase signaling regulates transforming growth factor- $\beta 1$-induced chondrogenesis and actin organization of synovium-derived mesenchymal stem cells through interaction with the Smad pathway
}

\author{
TING XU ${ }^{1}$, MENGJIE WU ${ }^{1}$, JIANYING FENG $^{2}$, XINPING LIN $^{2}$ and ZHIYUAN GU ${ }^{1,2}$ \\ ${ }^{1}$ Department of Oral and Maxillofacial Surgery, The Affiliated Hospital of Stomatology, Zhejiang University, Hangzhou, \\ Zhejiang 310006; ${ }^{2}$ School of Stomatology, Zhejiang Chinese Medical University, Hangzhou, Zhejiang 310053, P.R. China
}

Received April 8, 2012; Accepted May 28, 2012

DOI: $10.3892 /$ ijmm.2012.1107

\begin{abstract}
Recent studies have suggested that synoviumderived mesenchymal stem cells (SMSCs) may be promising candidates for tissue engineering and play an important role in cartilage regeneration. However, the mechanisms of SMSC chondrogenesis remain to be identified and characterized. The aim of this study was to evaluate the activation of the RhoA/ Rho kinase (ROCK) pathway, as well as the manner by which it may contribute to chondrogenesis and the actin cytoskeletal organization of rat temporomandibular SMSCs in response to transforming growth factor- $\beta 1$ (TGF- $\beta 1)$. Primary isolated SMSCs were treated with TGF- $\beta 1$, and their actin organization was examined by fluorescein isothiocyanate-phalloidin staining. The specific biochemical inhibitors, C3 transferase, Y27632 and SB431542, were employed to evaluate the function of RhoA/ROCK and Smads. The effect of C3 transferase and Y27632 on the gene expression of chondrocyte-specific markers was evaluated by quantitative real-time polymerase chain reaction. To examine the effect of Y27632 on Smad2/3 phosphorylation induced by TGF- $\beta 1$, western blot analysis was also performed. The stimulation of TGF- $\beta 1$ in SMSCs resulted in the activation of the RhoA/ROCK pathway and concomitantly induced cytoskeletal reorganization, which was specifically blocked by C3 transferase and Y27632. The TGF- $\beta$-induced gene expression of Sox9, type I collagen, type II collagen and aggrecan was also inhibited by both $\mathrm{C} 3$ transferase and Y27632, at different levels. Y27632 treatment reduced the phosphorylation of Smad2/3 in a concentration-dependent manner. These results demonstrate the RhoA/ROCK activation
\end{abstract}

Correspondence to: Dr Zhiyuan Gu, Department of Oral and Maxillofacial Surgery, The Affiliated Hospital of Stomatology, Zhejiang University, 395 Yan'an Road, Hangzhou, Zhejiang 310006, P.R. China

E-mail: gzy@zju.edu.cn

Key words: RhoA, transforming growth factor- $\beta 1$, Smad, synoviumderived mesenchymal stem cells, chondrogenesis regulates chondrocyte-specific gene transcription and cytoskeletal organization induced by TGF- $\beta 1$ by interacting with the Smad pathway. This may have significant implications for the successful utilization of SMSCs as a cell source for articular cartilage tissue engineering.

\section{Introduction}

Temporomandibular joint disorders (TMD), including temporomandibular joint osteoarthrosis (TMJ-OA), are a group of multifactorial joint diseases characterized by progressive joint degeneration $(1,2)$. Despite intensive research efforts, many challenges still remain in the treatment of TMJ-OA. The specific non-vascular property of the condylar cartilage makes self-repairing and regeneration difficult. Over the last decade, synovium-derived mesenchymal stem cells (SMSCs) have evoked interest as a very promising cell source for cartilage regeneration due to their superiority in proliferation and chondrogenesis (3-5). Previous studies have shown that SMSCs may be induced to undergo chondrogenesis when exposed to certain growth factors in vitro $(6,7)$. Although there has been great interest in the use of SMSCs for articular cartilage tissue engineering, the detailed mechanism of the chondrogenic differentiation of SMSCs remains unclear.

It is well established that the reorganization of the actin cytoskeleton is one of the earliest cellular responses to extracellular stimuli (8). During chondrogenesis, cells undergo changes in cell shape from a characteristic fibroblast-like structure to a round or polygonal morphology; these changes accompany the onset of gene expression levels, such as those of type II collagen and aggrecan (9-11). Therefore, the actin cytoskeleton appears to act as an integrated signaling network that plays a crucial role in regulating the differentiation and function of SMSCs. The transforming growth factor- $\beta 1$ (TGF- $\beta 1$ ), a member of the TGF- $\beta$ superfamily, is known to modulate diverse cellular responses, such as cell growth, proliferation, differentiation and production of the extracellular matrix. TGF- $\beta 1$ is the most extensively examined growth factor for inducing chondrogenesis and cartilage matrix synthesis $(7,12)$. Critical steps in the intracellular TGF- $\beta 1$ signaling pathway are mediated by different intracellular pathways, including the canonical Smad 
and non-Smad pathways (13). The signals are transduced by Smad and non-Smad pathways to the nucleus, where they regulate the expression of genes involved in chondrogenesis $(14,15)$. Moreover, TGF- $\beta 1$ has been shown to modulate cell growth and morphology in a concerted manner via a mechanism that controls the actin cytoskeleton in a number of cell types (16).

The Rho family small GTPases, including RhoA, Rac1 and $\mathrm{Cdc} 42$, are the best characterized upstream regulators of the actin cytoskeleton (17). The activation of Rho GTPases also controls a number of cellular processes, such as cell proliferation, cell cycle progression and gene expression, through a multitude of effector proteins $(18,19)$. The Rho kinases (ROCK)1 and ROCK2 are the most important downstream effectors of RhoA. Certain studies have shown that RhoA activity negatively affects chondrogenesis in the ATDC5 chondrogenic cell line and mesenchymal cells $(20,21)$. On the contrary, the addition of the ROCK inhibitor, Y27632, to micromass cultures of ATDC5 cells has been shown to inhibit chondrocyte-specific gene expression (22). In addition, the RhoA/ROCK pathway has been implicated in the TGF- $\beta 1$ matrix responses in various cells $(23,24)$. However, to the best of our knowledge, there has been no other report investigating the role of the RhoA/ROCK signaling pathway in the process of chondrogenesis in SMSCs.

The aim of the present study was to evaluate the activation of the RhoA/ROCK pathway in the TGF- $\beta 1$-induced chondrogenesis and actin organization of SMSCs, and to further investigate any interactions between the RhoA/ROCK and Smad pathways.

\section{Materials and methods}

Cell culture and treatment. SMSCs were isolated and expanded as reported by Li et al (25). Briefly, synovial membrane tissues were harvested from the temporomandibular joints of male Sprague-Dawley rats aged 6 weeks in accordance with the guidelines approved by the Animal Committee of the Zhejiang University. The excised tissues were vigorously washed in phosphate-buffered saline (PBS), and then minced into $1 \mathrm{~mm}^{3}$ pieces and plated in a T25 culture flask with a medium consisting of high-glucose Dulbecco's modified Eagle's medium (DMEM; Gibco-BRL, Gland Island, NY, USA) supplemented with 10\% fetal bovine serum (FBS; Gibco-BRL), penicillin (100 U/ml), streptomycin $(100 \mu \mathrm{g} / \mathrm{ml})$ and $4 \mathrm{mM} \mathrm{L}$-glutamine at $37^{\circ} \mathrm{C}$ with $5 \% \mathrm{CO}_{2}$. The cells were expanded in a monolayer culture for 3-5 passages. In order to evaluate SMSC chondrogensis, cells were seeded at a density of $5 \times 10^{4}$ cells/ $/ \mathrm{cm}^{2}$ in 6 -well plates and cultured in chondrogenic medium composed of high glucose DMEM supplemented with $100 \mathrm{nmol} / \mathrm{l}$ dexamethasone, $50 \mathrm{mg} / \mathrm{l}$ vitamin C, $40 \mu \mathrm{g} / \mathrm{ml}$ proline, $50 \mu \mathrm{g} / \mathrm{ml}$ ascorbate-2-phosphate, $100 \mu \mathrm{g} / \mathrm{ml}$ pyruvate (Sigma, St. Louis, MO, USA) and $10 \mathrm{ng} / \mathrm{ml}$ TGF- $\beta 1$ (R\&D Systems, Minneapolis, USA), replaced with each medium change. The culture medium was refreshed once every 2 days until day 7 . The cells that were cultured in chondrogenic medium without TGF- $\beta 1$ served as the control. Each experiment was repeated at least 3 times in order to verify the results.

Pharmacological inhibition study. A pharmacological inhibition study was subsequently performed to investigate the role of the RhoA/ROCK and Smad signaling pathways in
TGF- $\beta 1$-stimulated chondrogenesis. Specific inhibitors of RhoA (C3 transferase, $1 \mu \mathrm{M}$ ), ROCK (Y27632, 5 and $10 \mu \mathrm{M}$ ) (Cytoskeleton, Inc., Denver, CO, USA) and TGF- $\beta$ type I receptor (SB431542, 5 and $10 \mu \mathrm{M}$ ) (Tocris Bioscience, Bristol, UK) were added into the chondrogenic medium. The inhibitorcontaining chondrogenic medium was replenished once every 2 days.

RhoA activation assay. RhoA activation by TGF- $\beta 1$ was quantified using a luminescence based G-LISA RhoA activation assay biochemistry kit (Cytoskeleton). Immediately following chondrogenic stimulation in the presence of TGF- $\beta 1$ for different periods of time, the cells were rinsed with ice-cold PBS, and lysed using the provided cell lysis buffer. The lysates were then clarified by centrifugation at $10,000 \mathrm{rpm}$ at $4^{\circ} \mathrm{C}$ for $2 \mathrm{~min}$. After equalizing protein concentrations in all lysed cell extracts, GTP-bound RhoA levels were determined according to the manufacturer's instructions. Luminescence was detected as suggested by the manufacturer using a microplate spectrophotometer. The results were expressed relative to the untreated controls.

F-actin staining and fluorescent intensity. For the immunofluorescence detection of the actin cytoskeleton, the cells were grown on coverslips in 24-well plates, and then incubated with or without inhibitors of RhoA/ROCK ( $1 \mu \mathrm{M} \mathrm{C} 3$ transferase, $10 \mu \mathrm{M}$ Y27632) and Smads (10 $\mu \mathrm{M}$ SB431542) in the presence of TGF- $\beta 1$. The cells were rinsed with PBS and fixed in $4 \%$ paraformaldehyde for $30 \mathrm{~min}$. For F-actin staining, each sample was stained with fluorescein isothiocyanate (FITC)-phalloidin (Sigma) for $40 \mathrm{~min}$ at room temperature. Representative fluorescence images were captured using a fluorescence microscope (CX-RFL-2; Olympus, Tokyo, Japan) at x400 magnification. The average relative intensity of F-actin/cell in each group was analyzed using the MetaMorph software (Universal Imaging Corporation, Downingtown, PA, USA).

Protein isolation and western blot analysis. Total cell lysates were suspended in radio-immunoprecipitation assay lysis buffer (Beyotime, Shanghai, China) containing $1 \mathrm{mM}$ protease inhibitor phenylmethanesulfonyl fluoride (Beyotime) and incubated on ice for $30 \mathrm{~min}$. The samples were clarified by centrifugation at $12,000 \mathrm{rpm}$ for $5 \mathrm{~min}$ at $4^{\circ} \mathrm{C}$ and boiled for $5 \mathrm{~min}$ with a sample loading buffer. The protein concentrations were determined using a commercial bicinchoninic acid protein assay kit with bovine serum albumin as the standard. Equivalent protein amounts were fractionated by $10 \%$ sodium dodecyl sulfatepolyacrylamide gels and transferred onto $0.1 \mu \mathrm{M}$ polyvinylidene fluoride membranes (Millipore, Bedford, MA, USA). Blots were blocked with $5 \%$ non-fat milk for $1 \mathrm{~h}$, followed by incubation with specific primary antibodies against phosphorylated (phospho)-Smad2, phospho-Smad3, Smad2/3 and GAPDH (diluted in 1:1,000; Cell Signaling Technology, Inc., Danvers, MA, USA) overnight at $4^{\circ} \mathrm{C}$. After washing, the blots were then hybridized with specific horseradish peroxidase-conjugated secondary antibodies and visualized using an electrochemiluminescence reagent (Pierce, Rockford, IL, USA).

RNA extraction and cDNA synthesis. Total RNA was obtained using TRIzol reagent (Invitrogen Life Technologies, Carlsbad, CA, USA) according to the manufacturer's instructions. 
Table I. Primer sequences used during quantitative real-time RT-PCR analysis.

\begin{tabular}{lcl}
\hline Target gene & GenBank accession no. & Primer sequence $\left(5^{\prime} \rightarrow 3^{\prime}\right)$ \\
\hline ROCK1 & NM_031098.1 & Forward: AGAGGCTCAAGACATGCTCAATCA \\
ROCK2 & NM_013022.1 & Reverse: CAGTTAGCCGCGCTTTGGTTA \\
Type I collagen & & Forward: GTTCAGTTGGTTCGTCATA \\
Type II collagen & NM_053356.1 & Reverse: ATCATAATTGCTCATCAGGTTA \\
& & Forward: TGCTGGCCAACCATCCCTCT \\
Aggrecan & NM_012929.1 & Reverse: CGACATCATTGGATCCTTGCAG \\
Sox9 & NM_022190.1 & Forward: AGCGGAGACTACTGGATTGATC \\
& & Reverse: CTCTCCAAACCAGATGTGCTTC \\
GAPDH & XM_001081628.2 & Forward: AGCCATAGCTTCTCCTGAG \\
& & Reverse: GGGTATCTGACAGTCTGGTC \\
& NM_017008.3 & Forward: GAAGAGCAATGGTGACAGAG \\
& & Reverse: TGGAATCTCAGCAATCGTTAC \\
& & Forward: GAAGGTGAAGGTCGGAGTCG \\
\end{tabular}

The purity and yield of the isolated RNA were monitored using a NanoDrop ND-2000 Spectrophotometer (NanoDrop Technologies, Wilmington, DE, USA). The first-strand cDNA was synthesized from $1 \mu \mathrm{g}$ total RNA by reverse transcription using a SYBR PrimeScript ${ }^{\mathrm{TM}}$ RT reagent kit (Takara Bio, Inc., Dalian, China). The samples were diluted in nuclease-free water and stored at $-20^{\circ} \mathrm{C}$ prior to quantitative real-time polymerase chain reaction $(\mathrm{PCR})$.

Quantitative real-time PCR analysis. Quantitative real-time RT-PCR was performed using a SYBR PrimeScript ${ }^{\mathrm{TM}}$ RT-PCR kit (Takara) in the ABI PRISM 7500 Real-Time PCR System (Applied Biosystems, Foster City, CA, USA). The sequencespecific primers used for PCR amplification are listed in Table I. The thermal cycling conditions were 1 cycle at $95^{\circ} \mathrm{C}$ for $30 \mathrm{sec}, 40$ cycles at $95^{\circ} \mathrm{C}$ for $5 \mathrm{sec}$ and $60^{\circ} \mathrm{C}$ for $34 \mathrm{sec}$. The melting curve analysis for each PCR reaction was generated to ensure the purity of the amplified product (data not shown). Each gene was normalized against the corresponding glyceraldehyde-3-phosphate dehydrogenase (GAPDH) levels using the $\Delta \Delta \mathrm{CT}$ method (normalized $\mathrm{C}_{\mathrm{T}}$ values were expressed relative to the untreated controls) (26). The relative gene expression of each sample was shown.

Statistical analysis. All data were presented as the means \pm SD. The statistical analysis of the data was performed using the SPSS 19.0 software package by one-way ANOVA. Post hoc comparisons were made using Bonferroni corrections. $\mathrm{P}<0.05$ was considered to indicate a statistically significant difference.

\section{Results}

TGF- $\beta 1$-induces RhoA/ROCK activation in SMSCs. TGF- $\beta 1$ addition caused a rapid increase in RhoA activation after 1 day of stimulation, reaching its peak on day 4 , but gradually decreased as the time proceeded (Fig. 1A). RhoA activation leads to the activation of Rho kinase family members. Thus, these downstream effector kinases were assessed by quantitative real-time PCR analysis with emphasis on determining ROCK1 and ROCK2 mRNA expression. The treatment of SMSCs with TGF- $\beta 1$ resulted in an upregulation of the ROCK1 and ROCK2 genes (Fig. 1B and C). The expression of these genes was low on day 1 , but increased as chondrogenesis proceeded, peaked after 5 days, and was sustained at relatively high levels until day 7 . These results demonstrate that TGF- $\beta 1$ induces the activation of the RhoA/ROCK pathway in SMSCs.

TGF- $\beta 1$ causes RhoA/ROCK-dependent cytoskeletal responses in SMSCs. To identify the actin cytoskeletal characteristics of SMSCs, cells were treated with or without pharmacological inhibitors (see above) in addition to TGF- $\beta 1$ and visualized by fluorescence microscopy using FITC-phalloidin staining. After 7 days of in vitro expansion, the untreated control cells exhibited a relatively weak cytoplasmic staining of F-actin fibers, which were arranged randomly (Fig. 2). Compared with the control cells, TGF- $\beta 1$-stimulated cells showed a dramatically increased cytoplasmic staining of F-actin fibers, primarily arranged in parallel. The value expressed as an arbitrary unit also showed a dramatic increase of F-actin staining over the controls, indicating more filamentous actin formation (Fig. 3).

The cells that were treated with RhoA/ROCK inhibitors (1 $\mu \mathrm{M} \mathrm{C} 3$ transferase or $10 \mu \mathrm{M}$ Y27632) displayed a significant decrease in fluorescence intensity in comparison to TGF- $\beta 1$, indicating a reduced number of actin fibers. By contrast, treatment with T $\beta$ RI inhibitors (10 $\mu \mathrm{M}$ SB431542) did not lead to further alterations in actin fiber formation induced by TGF- $\beta 1$, showing no apparent change in the F-actin intensity of the SB431542 group. This finding demonstrates that RhoA/ ROCK activation plays a specific role in the control of TGF- $\beta 1$ induced actin cytoskeletal reorganization.

Involvement of RhoA signaling in TGF- $\beta 1$-induced chondrogenesis in SMSCs. By quantitative real-time PCR analysis, the chondrogenic potential of SMSCs was confirmed by assessing 

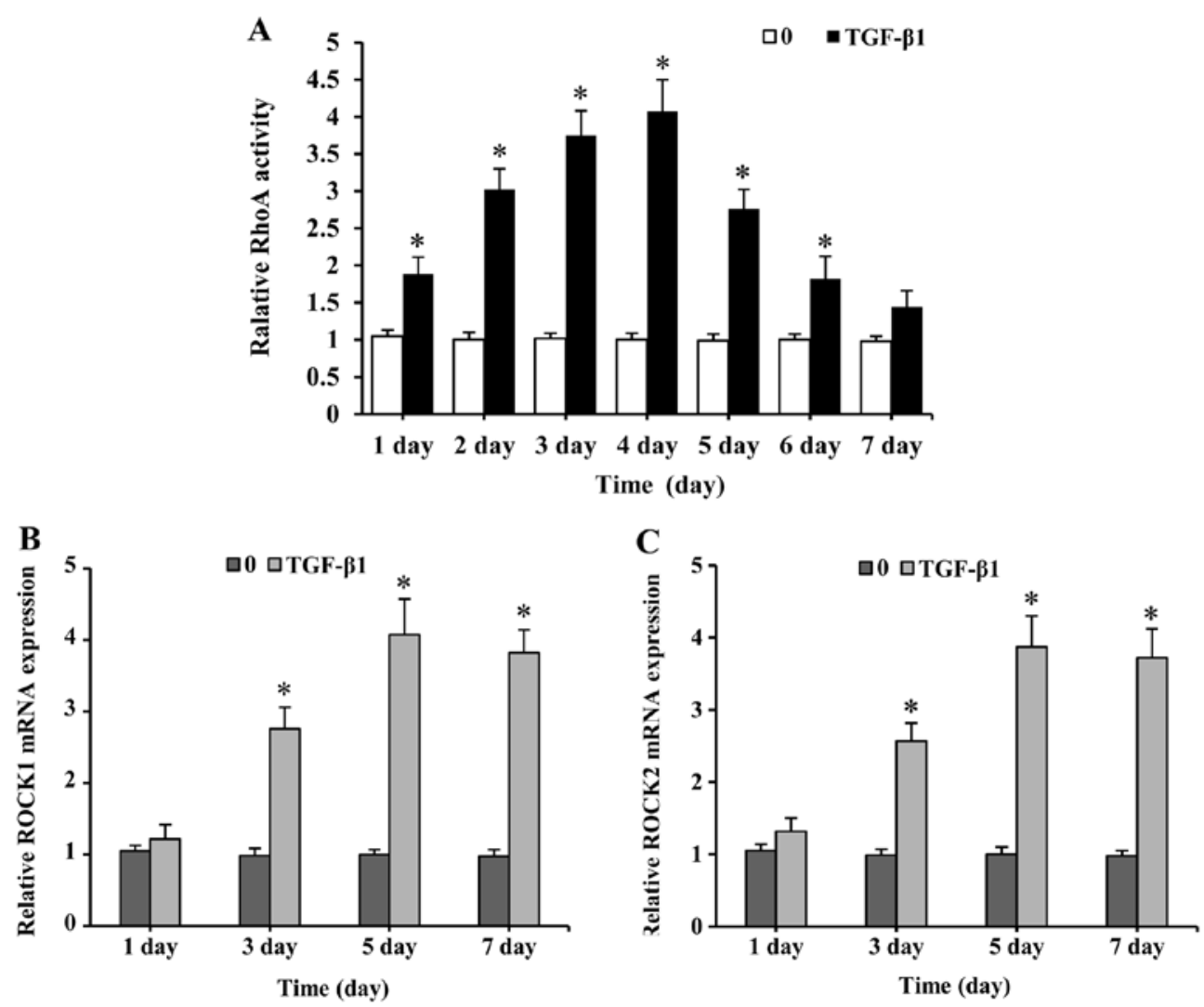

Figure 1. TGF- $\beta 1$-induced RhoA/ROCK activation in SMSCs. (A) Cell lysates were prepared at various time-points, and the levels of active GTP-bound RhoA were determined by a RhoA G-LISA kit. The relative levels of GTP-RhoA rapidly increased after 1 day of TGF- $\beta 1$ treatment, and decreased after 4 days. Quantitative real-time PCR analysis showed that the relative mRNA levels of (B) ROCK1 and (C) ROCK2 were low on day 1, peaked after 5 days, and sustained at relatively high levels until day 7 . Data are presented as the means $\pm \mathrm{SD}$, for $\mathrm{n}=6$ samples. * Significant difference compared to the untreated controls $(\mathrm{P}<0.05$ ).
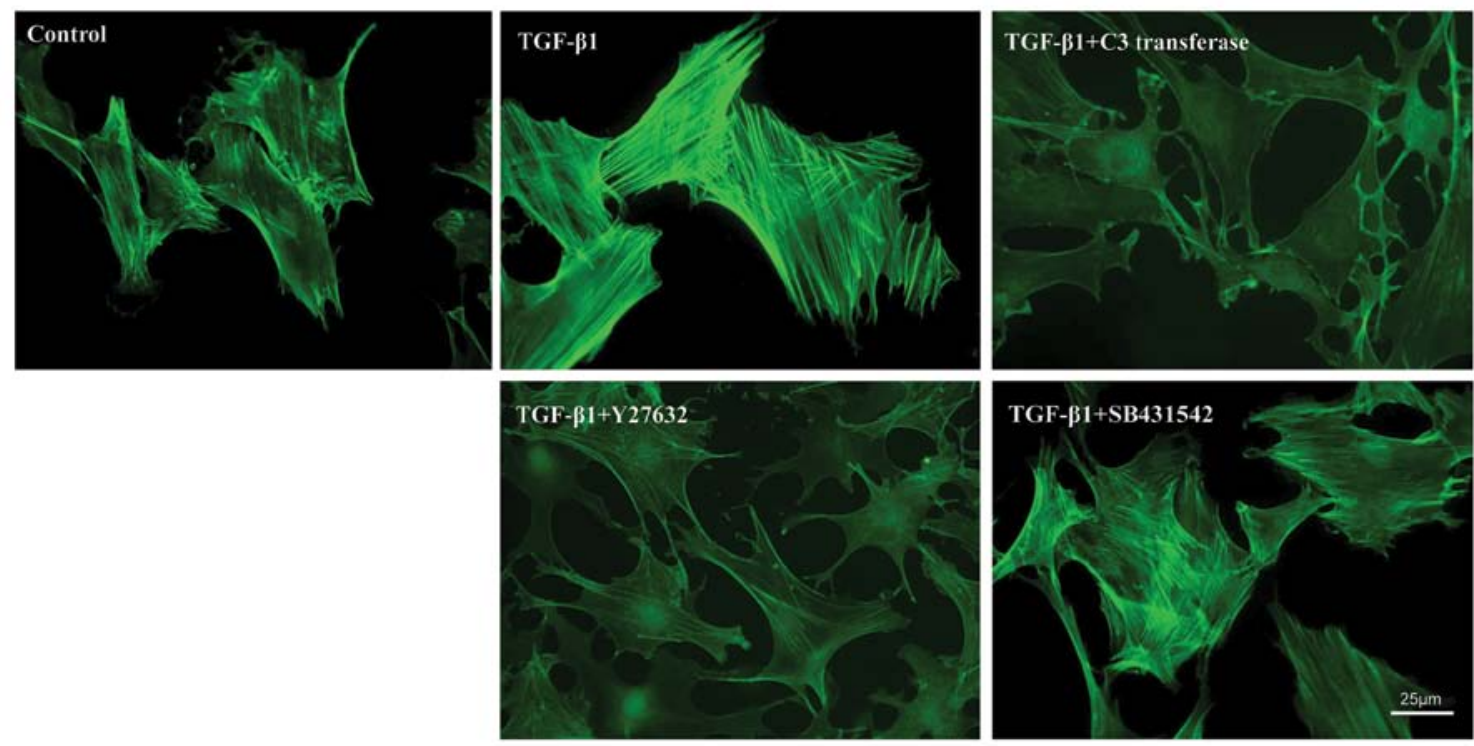

Figure 2. TGF- $\beta 1$ causes RhoA/ROCK-dependent cytoskeletal responses in SMSCs. Cells were treated with or without RhoA/ROCK (1 $\mu \mathrm{M} \mathrm{C} 3$ transferase, $10 \mu \mathrm{M}$ Y27632) and Smad (10 $\mu \mathrm{M}$ SB431542) inhibitors in the presence of TGF- $\beta 1$ for 7 days. All samples were stained with FITC-phalloidin (green signal) and imaged by fluorescence microscopy. Representative images are shown. Scale bar, $25 \mu \mathrm{m}$.

the chondrocyte-specific gene expressions of type I collagen, type II collagen and aggrecan. The mRNA levels of type I collagen, type II collagen and aggrecan significantly increased 7 days after the TGF- $\beta 1$ treatment compared with the untreated controls (Fig. 4A-C). Further treatment with the ROCK inhibitors resulted in a significantly reduced transcription of the cartilage-specific genes. Specifically, TGF- $\beta 1$-induced type I collagen, type II collagen and aggrecan mRNA levels 


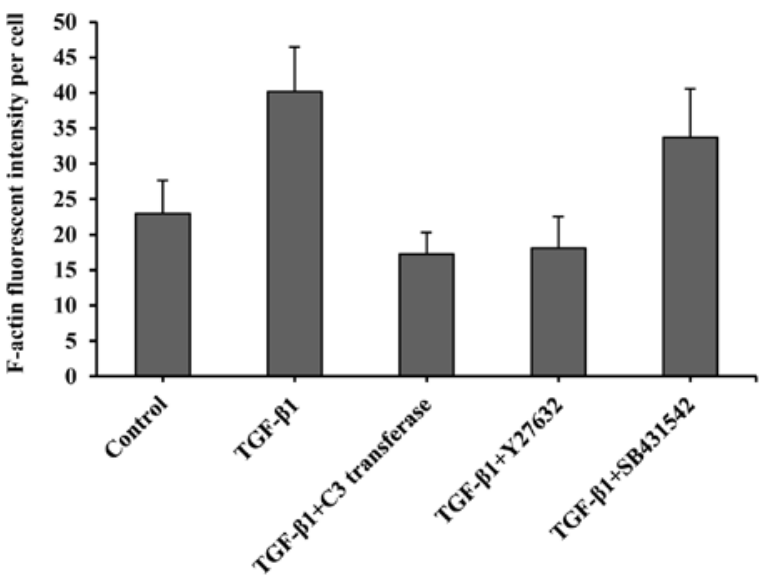

Figure 3. Average fluorescence intensity of F-actin (in arbitrary units) determined by fluorescence microscopy. Data are presented as the means \pm SD, for $n=6$ samples.

decreased by 30,44 and $51 \%$, respectively, after treatment with $1 \mu \mathrm{M} \mathrm{C} 3$ transferase. When treated with $10 \mu \mathrm{M}$ Y27632, the TGF- $\beta 1$-induced gene expressions levels of type I collagen, type II collagen and aggrecan were reduced by 37,59 and $49 \%$, respectively.

Sox 9 has been shown to be a major transcription regulator in the progression of chondrogenesis. Correlating the above observations in type I collagen, type II collagen and aggrecan expression levels, Sox 9 mRNA levels were significantly upregulated in the presence of TGF- $\beta 1$ (Fig. 4D). The induction of the Sox 9 mRNA expression was also repressed by C3 transferase and Y27632. The gene expression levels of type I collagen, type II collagen, aggrecan and Sox9 were also affected by $\mathrm{C} 3$ transferase and Y27632 in the absence of TGF- $\beta 1$. However, the influence of these inhibitors on gene expression was relatively minor compared with the samples cultured with TGF- $\beta 1$. These results suggest the involvement of RhoA/ROCK signaling in the regulation of TGF- $\beta 1$-induced chondrocyte-specific gene expression in SMSCs.

Effect of ROCK inhibitor on phosphorylation activity of Smads induced by TGF- $\beta 1$. To obtain insight into the possible interaction between RhoA/ROCK and TGF- $\beta 1$ signaling cascades, ROCK activation was specifically blocked with Y27632 at the final concentrations of 5 and $10 \mu \mathrm{M}$. Relatively high levels of phospho-Smad2 and phospho-Smad3 were maintained after 7 days of TGF- $\beta 1$ treatment (Fig. 5). After adding $5 \mu \mathrm{M}$ SB431542 to the TGF- $\beta 1$ group, the Smad2 and Smad3 phosphorylation levels dramatically decreased. When Y27632 was used at $5 \mu \mathrm{M}$, the TGF- $\beta 1$-induced phosphorylation of Smad2 and Smad3 in SMSCs was slightly reduced. In addition, treatment with $10 \mu \mathrm{M}$ Y27632 together with TGF- $\beta 1$ resulted in a marked reduction of Smad2 and Smad3 phosphorylation compared with the TGF- $\beta 1$ group, which indicated effective inhibition by this pharmacological inhibitor.

\section{Discussion}

SMSCs have previously been shown to have remarkable potential to undergo chondrogenic differentiation and to contribute toward tissue engineering and cartilage repair $(3,7)$.
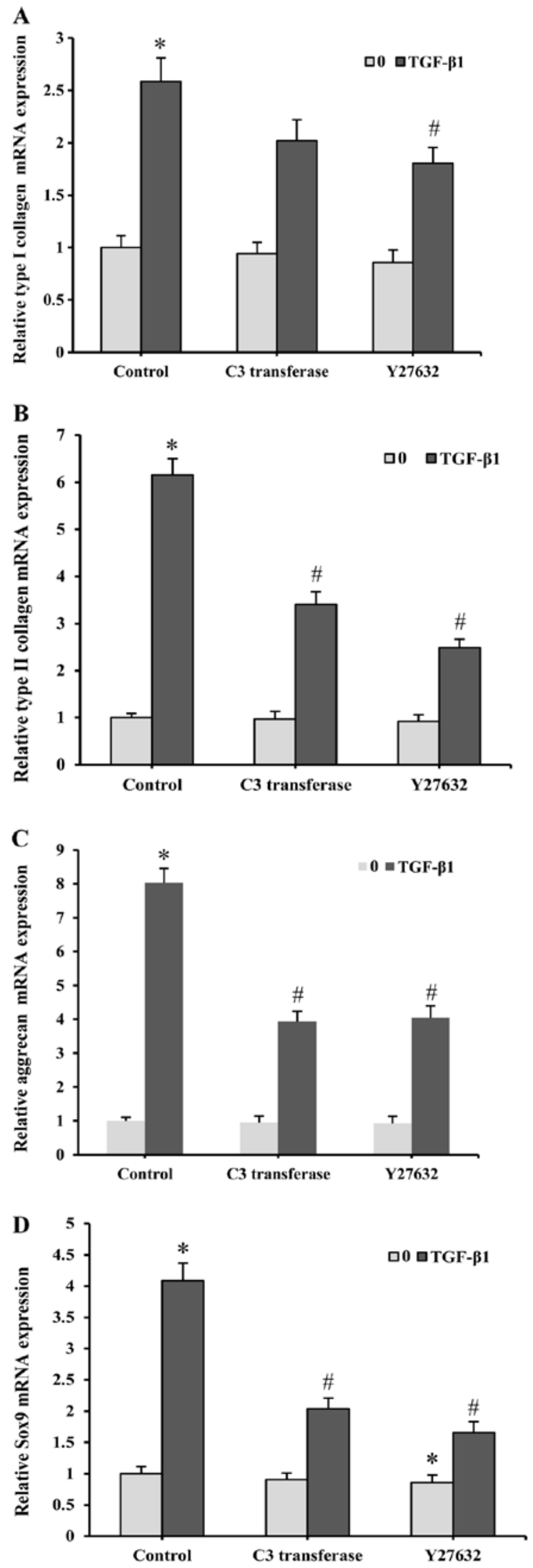

Figure 4. Effects of RhoA/ROCK inhibitors on TGF- $\beta 1$-induced (A) type I collagen, (B) type II collagen, (C) aggrecan and (D) Sox 9 mRNA expression levels. Quantitative real-time PCR was performed using RNA samples, which were isolated from SMSCs incubated with TGF- $\beta 1$ for 7 days in the presence or absence of C3 transferase $(1 \mu \mathrm{M})$ or Y27632 $(10 \mu \mathrm{M})$. Data are presented as the means $\pm \mathrm{SD}$, for $\mathrm{n}=6$ samples. *Significant difference compared to the

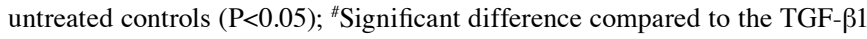
without inhibitor groups $(\mathrm{P}<0.05)$. 

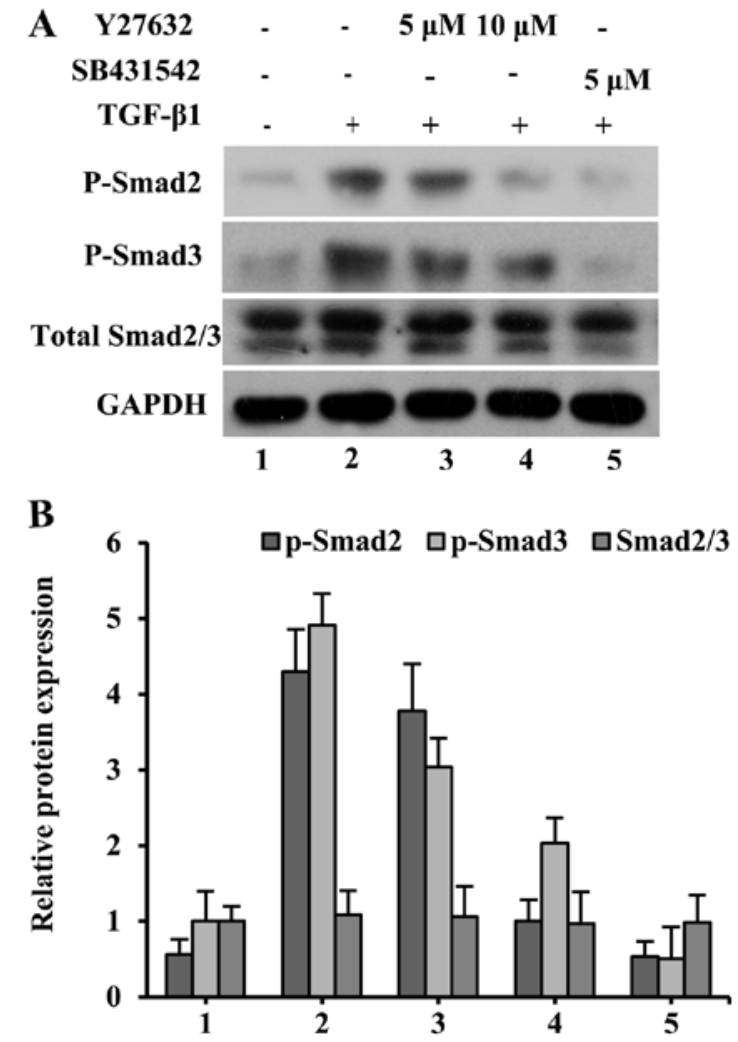

Figure 5. Effect of the ROCK inhibitor on the phosphorylation activity of Smads induced by TGF- $\beta 1$. Cells were treated with or without ROCK (Y27632, 5 or $10 \mu \mathrm{M}$ ) or Smad (SB431542, $5 \mu \mathrm{M}$ ) inhibitors in the presence of TGF- $\beta 1$ for 7 days. Total and phosphorylated Smad2/3 proteins were detected by western blot analysis in SMSCs. Blots are representative of 3 independent experiments

In the present study, the chondrogenic induction of SMSCs by TGF- $\beta 1$ was initially performed in vitro in a monolayer culture. Our study demonstrates the regulation of the RhoA/ROCK pathway and its interaction with the Smad signaling pathway during chondrogenic differentiation in response to TGF- $\beta 1$.

TGF- $\beta 1$ is a well-documented potent chondrogenic factor, which may induce extracellular matrix synthesis associated with cartilage regeneration $(7,12)$. The present results indicated that TGF- $\beta 1$ promoted chondrogenesis, as evidenced by the increased gene expression levels of type I collagen, type II collagen, aggrecan and Sox9. Concomitant to these enhanced expression levels, TGF- $\beta 1$-stimulated cells exhibited strong cytoplasmic actin fiber formation. Image analysis of FITCphalloidin stained SMSCs showed that TGF- $\beta 1$ treatment caused a dramatic increase in fluorescent intensity compared with the untreated controls. Therefore, TGF- $\beta 1$ induces a detailed phenotypic modification of SMSCs. However, the intracellular signaling pathways that mediate the functions and differentiation status of SMSCs by altering the actin cytoskeleton warrant further investigation.

Changes in the actin cytoskeleton may regulate cellular responses to TGF- $\beta 1$ by changes in receptor expression, focal adhesion signaling and nuclear signaling, which may alter cell differentiation and function (16). Over the last decade, RhoA signaling through the ROCK1/2 kinases has been implicated in regulating the shape-dependent control of mesenchymal cell differentiation via intrinsic mechanisms $(20,21,27)$. In this study, the TGF- $\beta 1$ stimulation of SMSCs was found to upregu- late RhoA activity rapidly in the early stage of chondrogenesis, but gradually decreased after 4 days. The upregulation of the gene expression of its downstream effectors, namely, ROCK1 and ROCK2, was also activated and sustained at a relatively higher level. To the best of our knowledge, this is the first study to show that RhoA activation, which may result in actin cytoskeleton reorganization, is also initiated by extrinsic growth factors and plays a significant role in the chondrogenic differentiation of SMSCs.

Notably, the results from our study demonstrate that type I collagen, type II collagen and aggrecan gene expression levels are significantly reduced by the inhibition of RhoA and ROCK activity with C3 transferase and Y27632, respectively. These findings suggest that the TGF- $\beta 1$-induced RhoA/ROCK activation plays a positive role in the chondrogenesis of SMSCs. When the RhoA/ROCK inhibitors were added, the TGF- $\beta 1$ induced cytoskeletal reorganization was interrupted, and chondrocyte-specific genes were downregulated. This suggests that chondrogenic differentiation requires an organized actin network that may be achieved by biochemically inducing RhoA activation. Additionally, the increasing cytoplasmic stress fiber formation is associated with the downstream target transcription of the chondrocyte-specific gene.

Sox9 is a key transcription factor in chondrogenic differentiation, and its expression directly activates the transcription of collagen II and aggrecan genes (28). Thus, whether or not the upstream transcription factor, Sox 9, was affected by the inhibition of RhoA/ROCK signaling was further assessed. In the current study, treatment with C3 transferase or Y27632 was found to suppress the influence of TGF- $\beta 1$ on transcription factor Sox 9 mRNA levels. This result is in agreement with a previous observation by Haudenschild et al (29), demonstrating that ROCK directly phosphorylated Sox 9 at Ser181 and resulted in the enhancement of Sox 9 activity in chondrocytes. Woods and Beier (22) demonstrated that ROCK inhibition reduced the activity of the Sox9-responsive reporter gene during chondrogenesis, but it was not in cellular context (20). However, the specific transcription factors connecting RhoA/ROCK to the Sox 9 transcription have not been identified. The differences in these results may be due to the differences among cell types, chondrogenic culture conditions, and the presence of specific growth factors and cytokines. Therefore, there is a compelling need to examine the roles of RhoA and its effectors in vivo and to identifiy the upstream and downstream components.

Since both RhoA/ROCK and Smad pathways are known to mediate TGF- $\beta 1$ signaling during chondrogenesis, we investigated their potential interaction in chondrogenesis. A number of studies have attempted to link Smads with RhoA/ ROCK activity. In human breast cancer cells, RhoA activation by TGF- $\beta$ has been demonstrated to be independent of Smads (30), whereas various studies have conversely shown a potential regulation of Smad signaling by RhoA/ ROCK $(23,31)$. In this regard, we observed a decrease of TGF- $\beta$-induced Smad $2 / 3$ phosphorylation with an increasing concentration of Y2763, suggesting that Y2763 inhibits the activation of the TGF- $\beta /$ Smad signaling pathway. The regulatory effect of the RhoA/ROCK pathway on Smad activity was consistent with the chondrocyte-specific genes. Therefore, this study provides evidence that RhoA/ROCK mediates the chondrogenesis of SMSCs by interacting with the Smad2/3 
pathway. However, in addition to further investigation of alternative cytoskeletal organizations, such as microtubular and intermediate filaments, TGF- $\beta 1$ possibly initiates other signaling cascades involved in chondrogenesis. Therefore, further research is required to fully elucidate the interaction between RhoA/ROCK and other signaling pathways in TGF- $\beta 1$ stimulation.

In conclusion, to the best of our knowledge, we report for the first time that the RhoA/ROCK activation initiated by TGF- $\beta 1$ has great potential to regulate cytoskeletal organization, as well as control the chondrogenic gene expression of SMSCs by interacting with the TGF- $\beta /$ Smad signaling pathway. These findings provide novel insights into the regulatory mechanisms that define the chondrogenetic differentiation of SMSCs, making the RhoA/ROCK pathway an interesting therapeutic target for the successful use of SMSCs as a cell source for cartilage tissue engineering.

\section{Acknowledgements}

The present study was supported by grants from the National Natural Science Foundation of China (nos. 81170979 and 30901687), the Natural Science Foundation of Zhejiang Province (nos. Y2090262 and Y2080370), and the Young Scientist Project from the Health Bureau of Zhejiang (no. 2009QN018).

\section{References}

1. Scrivani SJ, Keith DA and Kaban LB: Temporomandibular disorders. N Engl J Med 359: 2693-2705, 2008.

2. Tanaka E, Detamore MS and Mercuri LG: Degenerative disorders of the temporomandibular joint: etiology, diagnosis, and treatment. J Dent Res 87: 296-307, 2008.

3. Fan J, Varshney RR, Ren L, Cai D and Wang DA: Synoviumderived mesenchymal stem cells: a new cell source for musculoskeletal regeneration. Tissue Eng Part B Rev 15: 75-86, 2009.

4. Sakaguchi Y, Sekiya I, Yagishita K and Muneta T: Comparison of human stem cells derived from various mesenchymal tissues: superiority of synovium as a cell source. Arthritis Rheum 52: 2521-2529, 2005.

5. Yoshimura H, Muneta T, Nimura A, Yokoyama A, Koga $\mathrm{H}$ and Sekiya I: Comparison of rat mesenchymal stem cells derived from bone marrow, synovium, periosteum, adipose tissue, and muscle. Cell Tissue Res 327: 449-462, 2007.

6. Sampat SR, O'Connell GD, Fong JV, Alegre-Aguaron E, Ateshian GA and Hung CT: Growth factor priming of synoviumderived stem cells for cartilage tissue engineering. Tissue Eng Part A 17: 2259-2265, 2011.

7. Pei M, He F and Vunjak-Novakovic G: Synovium-derived stem cell-based chondrogenesis. Differentiation 76: 1044-1056, 2008.

8. Papakonstanti EA and Stournaras C: Cell responses regulated by early reorganization of actin cytoskeleton. FEBS Lett 582: 2120-2127, 2008.

9. Beier F and Loeser RF: Biology and pathology of Rho GTPase, PI-3 kinase-Akt, and MAP kinase signaling pathways in chondrocytes. J Cell Biochem 110: 573-580, 2010.

10. Woods A, Wang G and Beier F: Regulation of chondrocyte differentiation by the actin cytoskeleton and adhesive interactions. J Cell Physiol 213: 1-8, 2007.
11. Blain EJ: Involvement of the cytoskeletal elements in articular cartilage homeostasis and pathology. Int J Exp Pathol 90: 1-15, 2009.

12. Yamane $\mathrm{S}$ and Reddi $\mathrm{AH}$ : Induction of chondrogenesis and superficial zone protein accumulation in synovial side population cells by BMP-7 and TGF-beta1. J Orthop Res 26: 485-492, 2008.

13. Shi Y and Massague J: Mechanisms of TGF-beta signaling from cell membrane to the nucleus. Cell 113: 685-700, 2003.

14. McMahon LA, Prendergast PJ and Campbell VA: A comparison of the involvement of p38, ERK1/2 and PI3K in growth factorinduced chondrogenic differentiation of mesenchymal stem cells. Biochem Biophys Res Commun 368: 990-995, 2008.

15. Tuli R, Tuli S, Nandi S, Huang X, Manner PA and Hozack WJ, et al: Transforming growth factor-beta-mediated chondrogenesis of human mesenchymal progenitor cells involves $\mathrm{N}$-cadherin and mitogen-activated protein kinase and Wnt signaling cross-talk. J Biol Chem 278: 41227-41236, 2003.

16. Moustakas A and Heldin CH: Dynamic control of TGF-beta signaling and its links to the cytoskeleton. FEBS Lett 582: 2051-2065, 2008

17. Etienne-Manneville $S$ and Hall A: Rho GTPases in cell biology. Nature 420: 629-635, 2002.

18. Mammoto A, Huang S, Moore K, Oh P and Ingber DE: Role of RhoA, mDia, and ROCK in cell shape-dependent control of the Skp2-p27kip1 pathway and the G1/S transition. J Biol Chem 279: 26323-26330, 2004.

19. Wang G and Beier F: Rac1/Cdc42 and RhoA GTPases antagonistically regulate chondrocyte proliferation, hypertrophy, and apoptosis. J Bone Miner Res 20: 1022-1031, 2005.

20. Woods A, Wang G and Beier F: RhoA/ROCK signaling regulates Sox9 expression and actin organization during chondrogenesis. J Biol Chem 280: 11626-11634, 2005.

21. McBeath R, Pirone DM, Nelson CM, Bhadriraju K and Chen CS: Cell shape, cytoskeletal tension, and RhoA regulate stem cell lineage commitment. Dev Cell 6: 483-495, 2004.

22. Woods $A$ and Beier F: RhoA/ROCK signaling regulates chondrogenesis in a context-dependent manner. J Biol Chem 281: 13134-13140, 2006.

23. Hubchak SC, Runyan CE, Kreisberg JI and Schnaper HW: Cytoskeletal rearrangement and signal transduction in TGF-beta1-stimulated mesangial cell collagen accumulation. J Am Soc Nephrol 14: 1969-1980, 2003.

24. Wang S, Wu X, Lincoln TM and Murphy-Ullrich JE: Expression of constitutively active cGMP-dependent protein kinase prevents glucose stimulation of thrombospondin 1 expression and TGF-beta activity. Diabetes 52: 2144-2150, 2003.

25. Li J, Long X, Ke J, Meng QG and Fang W: Identification and characterization of synovial mesenchymal stem cells in temporomandibular joint. Zhonghua Kou Qiang Yi Xue Za Zhi 40: 362-364, 2005 (In Chinese).

26. Livak KJ and Schmittgen TD: Analysis of relative gene expression data using real-time quantitative PCR and the 2(-Delta Delta C(T)) method. Methods 25: 402-408, 2001.

27. Wang G, Woods A, Sabari S, Pagnotta L, Stanton LA and Beier F: RhoA/ROCK signaling suppresses hypertrophic chondrocyte differentiation. J Biol Chem 279: 13205-13214, 2004.

28. Bi W, Deng JM, Zhang Z, Behringer RR and de Crombrugghe B: Sox9 is required for cartilage formation. Nat Genet 22: 85-89, 1999.

29. Haudenschild DR, Chen J, Pang N, Lotz MK and D'Lima DD: Rho kinase-dependent activation of SOX9 in chondrocytes. Arthritis Rheum 62: 191-200, 2010.

30. Kamaraju AK and Roberts AB: Role of Rho/ROCK and p38 MAP kinase pathways in transforming growth factor-betamediated Smad-dependent growth inhibition of human breast carcinoma cells in vivo. J Biol Chem 280: 1024-1036, 2005.

31. Chen S, Crawford M, Day RM, Briones VR, Leader JE and Jose PA, et al: RhoA modulates Smad signaling during transforming growth factor-beta-induced smooth muscle differentiation. J Biol Chem 281: 1765-1770, 2006. 